\title{
Frequent injections of high-dose human umbilical cord mesenchymal stem cells slightly aggravate arthritis and skeletal muscle cachexia in collagen-induced arthritic mice
}

\author{
LEMEI AN $^{1 *}$, TIANSHU CHU ${ }^{1 *}$, LIUJUN WANG $^{1}$, SONGTAO AN $^{2}$, \\ YALONG $\mathrm{LI}^{3}$, HONGBO $\mathrm{HAO}^{4}$, ZHUOLI ZHANG ${ }^{5}$ and $\mathrm{HAN} \mathrm{YUE}^{3}$
}

\begin{abstract}
Departments of ${ }^{1}$ Rheumatology and Clinical Immunology and ${ }^{2}$ Cardiology; ${ }^{3}$ Henan Key Laboratory of Stem Cell Differentiation and Modification, Stem Cell Research Center, Henan Provincial People's Hospital, People's Hospital of Zhengzhou University, Zhengzhou, Henan 450003, P.R. China; ${ }^{4}$ Neuroscience Initiative, Advanced Science Research Center at the Graduate Center, City University of New York, New York, NY 10031, USA; ${ }^{5}$ Department of Rheumatology and Clinical Immunology, Peking University First Hospital, Beijing 100034, P.R. China
\end{abstract}

Received December 28, 2020; Accepted May 14, 2021

DOI: $10.3892 / \mathrm{etm} .2021 .10707$

\begin{abstract}
A single injection of low-dose human umbilical cord-derived mesenchymal stem cells (UC-MSCs) has been previously demonstrated to relieve synovitis and bone erosion in animal models of arthritis, but whether frequent injections of high-dose UC-MSCs relieve arthritis and inhibit loss of muscle mass has remained elusive. In the present study, DBA/1 mice were randomly divided into three groups: Normal (wild-type mice; $n=11)$, collagen-induced arthritis $(\mathrm{CIA} ; \mathrm{n}=12)$ and CIA treated with UC-MSCs $\left(\mathrm{n}=11 ; 5 \times 10^{6}\right.$ UC-MSCs per week for 3 weeks). Arthritis and skeletal muscle cachexia were evaluated until the end of the experiment on day 84. It was indicated that both the CIA and UC-MSC groups had lower body weights compared with the normal mice. Clinical arthritis scores, hind ankle diameters, synovitis and bone erosion progressively increased and were similar between the CIA and UC-MSC groups. Although there was no difference in food intake among the three groups, the normalized food intake of normal group was significantly higher than CIA group and UC-MSC group from day 42 onwards; there
\end{abstract}

Correspondence to: Professor Zhuoli Zhang, Department of Rheumatology and Clinical Immunology, Peking University First Hospital, 8 Xishiku Street West District, Beijing 100034, P.R. China E-mail: zhuoli.zhang@126.com

Dr Han Yue, Henan Key Laboratory of Stem Cell Differentiation and Modification, Stem Cell Research Center, Henan Provincial People's Hospital, People's Hospital of Zhengzhou University, 7 Weiwu Road, Zhengzhou, Henan 450003, P.R. China

E-mail: yuehan1000@126.com

*Contributed equally

Key words: rheumatoid arthritis, muscle cachexia, inflammation, fibrosis, human umbilical cord mesenchymal stem cells was no significance on day 77 but this could be neglected. Furthermore, gastrocnemius muscle weight in the UC-MSC group was significantly reduced compared with that in the CIA and normal groups. The UC-MSC group had higher levels of proinflammatory cytokines, such as TNF- $\alpha$, IL-6 and IL-1 $\beta$ than those in the CIA group. However, the other cytokines assessed and the fibrosis indices in the CIA and UC-MSC groups were not different from those in the control group and there was no inflammatory cell infiltration. Thus, frequent injections of high-dose UC-MSCs slightly aggravated synovitis and muscle cachexia in the murine CIA model and should therefore be avoided in the treatment of arthritis.

\section{Introduction}

Rheumatoid arthritis (RA) is an immune-mediated systemic inflammatory disease of unknown etiology, characterized by polyarticular synovial inflammation and bone erosion (1). Besides arthritis symptoms and other complications, 17-60\% of patients with RA experience rheumatoid cachexia (2). Cachexia is a serious complication of RA and other chronic illnesses, such as diabetes, cancer and heart failure (3). However, RA-related musculoskeletal alterations are frequently overlooked, despite their contributions to the physical disability and reduced mobility and quality of life of patients (4). Arthritis and rheumatoid cachexia may be modeled in collagen-induced arthritis (CIA) mouse models, which provides the possibility of studying the disease (5).

Although the outcomes of numerous patients with RA have been significantly improved by the wide use of conventional synthetic disease-modifying antirheumatic drugs (DMARDs), biological DMARDs and targeted synthetic DMARDs, a considerable proportion of patients with RA remain in non-remission or experience side effects from the drugs, which is mainly due to the incomplete understanding of the etiology and drug intolerance (6). Thus, advancing the understanding of RA to identify novel therapeutic strategies is critical for improving the efficacy of current treatments. 
Stem cell-based therapy has emerged as a promising approach to treat arthritic diseases. It was previously reported that transplantation of adult stem cells, such as bone marrow stem cells, adipose mesenchymal stem cells (MSCs) or umbilical cord-derived MSCs (UC-MSCs) into the blood was able to ameliorate synovitis and bone erosion in animal models of arthritis (7). Accumulating evidence has indicated the therapeutic potential of UC-MSCs in animal models of arthritis and clinical trials (8). These effects are systemic and integrated, suggesting that UC-MSCs may be a preferred resource to systematically improve synovitis and bone erosion. Compared with other types of stem cell, UC-MSCs are able to secrete a wide range of multifunctional factors and have been demonstrated to possess systemic immunoregulatory properties (9). Therefore, UC-MSCs may be considered more promising for clinical application compared with other types of stem cell.

However, there are certain emerging limitations that cannot be ignored. For instance, the therapeutic doses and frequencies remain critical issues that require to be determined $(10,11)$. Furthermore, whether frequent injections of high-dose UC-MSCs cause enhanced efficacy or aggravated adverse effects has remained elusive. In addition, muscle atrophy in patients with RA has received little attention in previous studies. Whether doses and frequencies of UC-MSC injections that differ from those applied previously are a more favorable choice for treating arthritis and cachexia also requires further research. Therefore, the present study aimed to investigate the effects of UC-MSCs on the joints and muscles of mice with CIA when administered frequently at high doses, hoping that the results provide novel insight into the therapeutic efficacy of UC-MSCs in RA-related muscle cachexia.

\section{Materials and methods}

Preparation of UC-MSCs. The UC was obtained from a 25-year-old healthy mother from March 28, 2019 at Henan Provincial People's Hospital (Zhengzhou, China) after cesarean deliveries. Written informed consent was obtained from the donor several weeks prior to delivery. The UC-MSCs were cultured, purified and identified as previously described (12). The viability of UC-MSCs digested by $0.2 \%$ Type I collagenase (cat. no. 17100017; Gibco; Thermo Fisher Scientific, Inc.) was $>95 \%$ (as assessed via trypan blue staining) and all cultures were negative for pathogenic microorganisms, including bacteria (on blood agar plates), fungi (in Sabouraud dextrose medium), endotoxins (as detected using a horseshoe crab agent) and mycoplasma [as determined via reverse transcription-PCR (RT-PCR)] (13). Flow cytometric analysis identified the presence of CD73, CD90 and CD105 (>95\% of cells) and the absence of CD34, CD11b, CD19, CD45 and human leukocyte antigen-DR using a Human MSC Analysis kit (BD Biosciences; cat. no. 562245) ( $<2 \%$ of cells) on the cell surface (14) (Fig. S1). All procedures for isolating, culturing and harvesting UC-MSCs were performed under good manufacturing practice conditions. All UC-MSCs used for transfusion were from passage 3 .

Mice and CIA model. The experiments were performed on 34 male DBA/1 mice (weight, 15-18 g; age, 10 weeks; Beijing Vital River Laboratory Animal Technology). All mice, with 4 mice housed per cage, were maintained in a specific pathogen-free environment on a standard chow and water with a $12 \mathrm{~h}$ dark-light cycles at $20 \pm 2^{\circ} \mathrm{C}$ and $55 \pm 10 \%$ humidity. The mice were randomly divided into three groups: Normal group $(n=11)$, CIA group $(n=12)$ and a CIA group treated with UC-MSCs $\left(n=11\right.$; dose, $5 \times 10^{6}$ per mouse every week for 3 weeks). CIA mice were established as described previously $(15,16)$. In brief, equal volumes of bovine type II collagen (Chondrex, Inc.) and complete Freund's adjuvant ( $4 \mathrm{mg} / \mathrm{ml}$; Chondrex, Inc.) were mixed to form an emulsion. On day $0,0.1 \mathrm{ml}$ emulsion was injected intradermally at a site $1.5 \mathrm{~cm}$ distal to the base of the tail. On day 21, the mice received an identical dose of type II collagen mixed with an equal volume of normal saline injected intraperitoneally. Normal mice were injected with $0.1 \mathrm{ml}$ normal saline on both above-mentioned occasions. The study protocol was approved by the Animal Ethics Committee of Peking University (Beijing, China; approval no. J201985). All experiments were performed in accordance with the National Institutes of Health Guide for the Care and Use of Laboratory Animals.

Experimental procedures. Locomotor function was assessed via an open field test (OFT) in mice with CIA when the arthritis clinical score reached 3 (15) at days 28 . Then, mice were assigned to the UC-MSC group received $5 \times 10^{6} \mathrm{UC}-\mathrm{MSC}$ on days 28, 35 and 42 (11), which were injected via the tail vein. No animals died unexpectedly prior to the end of this experiment. Body weight, food intake (measured as the weight of the remaining food pellets subtracted from the initial food weight), arthritis clinical score and hind ankle diameters measured using a caliper were evaluated each week (15). On day 84 (11), the mice were anesthetized via intraperitoneal injection of pentobarbital sodium at a concentration of $50 \mathrm{mg} / \mathrm{kg}$ and were sacrificed by cervical dislocation. The left gastrocnemius muscle and the knee joints of each mouse were dissected for histopathological analyses and the gastrocnemius muscle on the right side was stored at $-196^{\circ} \mathrm{C}$ in liquid nitrogen for RT-quantitative (q)PCR.

Open Field Test $(O F T)$. The mice were first familiarized with the square testing arena $(50 \times 50 \times 35 \mathrm{~cm})$ in a quiet, dimly lit room for $6 \mathrm{~min}(1 \mathrm{~min}$ for adapting to the environment and 5 min for the OFT). On days 0 and 28 , the mice were placed in the open field arena for $5 \mathrm{~min}$ and spontaneous locomotor activities were recorded using a video analysis system of OFT (BW-OF302, Shanghai Ranlong Technology Development Co., Ltd.) mounted on top of the arena for measurements of the total time spent active and immobile, the total movement distance and the mean velocity of motion (17).

Histology and scoring of arthritis. The knee joints from the hind limbs were excised and fixed in $10 \%$ buffered formalin for $24 \mathrm{~h}$ at room temperature. Subsequently, the samples were decalcified in $10 \%$ EDTA for 21 days at room temperature and embedded in paraffin. Slides with a thickness of $4 \mu \mathrm{m}$ were dewaxed with $100 \%$ xylene and then rehydrated with anhydrous, 95 and $70 \%$ ethanol and $\mathrm{H}_{2} \mathrm{O}$ in sequence. The slices were then stained with haematoxylin for $5 \mathrm{~min}$ and eosin for $1 \mathrm{~min}$ and then dehydrated at room temperature. All HE-stained slides were captured and analyzed using an 
Olympus BX53 light microscope (Olympus Corporation) at 20x magnification. Synovial inflammation was scored by two independent observers (LA and LW) according to the percentage area exhibiting synovitis in five high-power magnification fields per animal: 0, 0\% (absent); 1, 1-10\% (mild); 2, 11-50\% (moderate); and 3, 51-100\% (severe). Cartilage erosion was scored according to the percentage of the cartilage surface that was eroded: $0,0 \%$ (absent); $1,1-10 \%$ (mild); 2, 11-50\% (moderate); and 3, 51-100\% (severe). The absence of bone erosion was scored as 0 , minor erosion(s) observed only at high magnification was scored as 1 , moderate erosion(s) observed at low magnification was scored as 2 and severe transcortical erosion(s) was scored as 3 (18).

The gastrocnemius muscles from the hind limbs were excised and placed in a fixative solution (Wuhan Servicebio Technology Co., Ltd.) for $24 \mathrm{~h}$ before paraffin embedding, cut into $5 \mu \mathrm{m}$ sections and stained with $\mathrm{HE}$ at room temperature.

Myofiber cross-sectional area. Myofiber cross-sectional areas were calculated as described previously (18). In brief, fibers were measured in two randomly selected images of the gastrocnemius muscle from a series of 10 images per mouse, totaling 100-200 measured myofibers. The cross-sectional area of the gastrocnemius fibers was calculated using ImageJ software (version 1.8.0; National Institutes of Health) (19).

Toluidine blue staining. Toluidine Blue O Cartilage Stain solution containing an amine group and a quinone type benzene ring as chromogenic agents (cat. no. G2543; Beijing Solarbio Science \& Technology Co., Ltd.) was added to cartilage samples for $15 \mathrm{~min}$ at room temperature. Acetone $(1,000 \mathrm{ml})$ was then applied to differentiate chondrocytes for $5 \mathrm{~min}$ at room temperature.

Immunohistochemical staining. Gastrocnemius muscle tissues were fixed in muscle fixation fluid (cat. no. G1111; Wuhan Servicebio Technology Co., Ltd.) at room temperature for $24 \mathrm{~h}$, subsequently dehydrated and thereafter embedded in paraffin. Tissue sections $(4 \mu \mathrm{m})$ were deparaffinized according to the above-mentioned methods and incubated with $3 \% \mathrm{H}_{2} \mathrm{O}_{2}$ at $37^{\circ} \mathrm{C}$ for $10 \mathrm{~min}$. Antigen retrieval was performed by boiling in $0.01 \mathrm{M}$ sodium citrate at $95^{\circ} \mathrm{C}$ for $15 \mathrm{~min}$. After cooling for $2 \mathrm{~h}$, the sections were blocked at room temperature for $1 \mathrm{~h}$ using 10\% goat serum (z sbio.com, SAP-9102). The slides were then incubated at $4^{\circ} \mathrm{C}$ overnight with antibodies against CD3 (cat. no. GB13014; 1:100 dilution), CD4 (cat. no. GB11064; 1:500 dilution), CD19 (cat. no. GB11061-1; 1:300 dilution), F4/80 (cat. nos. GB11061-1 and GB11027; 1:1,500 dilution) and inducible nitric oxide synthase (iNOS; cat. no. GB11119; 1:2,000 dilution). Subsequently, the sections were incubated with biotinylated goat anti-rabbit horseradish peroxidase IgG secondary antibody (cat. no. G1213-100UL; 1:1,000 dilution) at $37^{\circ} \mathrm{C}$ for $30 \mathrm{~min}$. All antibodies were from Wuhan Servicebio Technology Co., Ltd. Diaminobenzidine was used as chromogenic agent. Following washing, the sections were counterstained with hematoxylin, dehydrated with ethanol $(70,85,95$ and $100 \%)$ and xylene and then sealed with neutral resin. Images were obtained using a light microscope (magnification, x400; Olympus Corporation).
$R T-q P C R$. Total RNA was isolated from gastrocnemius muscle using TRIzol ${ }^{\circledR}$ (Takara Bio, Inc.) and cDNA was synthesized at $37^{\circ} \mathrm{C}$ for $15 \mathrm{~min}$ and $85^{\circ} \mathrm{C}$ for $5 \mathrm{sec}$ using reverse transcriptase (PrimeScript RTase, RNase Inhibitor, Random 6 mers, Oligo dT Primer, dNTP Mixture, Buffer; cat. no. RR036A; Takara Bio, Inc.). The expression levels of genes encoding IL-6, IL-1 $\beta$, TNF- $\alpha$, iNOS, IL-10, $\alpha$-smooth muscle actin ( $\alpha$-SMA), fibronectin 1 (FN1), collagen type I $\alpha 1$ chain (COLIa1), COLIa2, COLIIIa1 and TGF- $\beta 1$ were assessed via RT-qPCR using SYBR green mix (Applied Biosystems; Thermo Fisher Scientific, Inc.) and the primers are listed in Table SI. The thermocycling conditions were as follows: Initial denaturation at $50^{\circ} \mathrm{C}$ for $2 \mathrm{~min}$ and $95^{\circ} \mathrm{C}$ for $2 \mathrm{~min}$, followed by 40 cycles of $95^{\circ} \mathrm{C}$ for $15 \mathrm{sec}, 60^{\circ} \mathrm{C}$ for $15 \mathrm{sec}$ and $72^{\circ} \mathrm{C}$ for $1 \mathrm{~min}$. Data were collected and quantitatively analysed on an ABI Prism 7900 sequence detection system (Applied Biosystems; Thermo Fisher Scientific, Inc.). Finally, relative gene expression values were calculated using the $2^{-\Delta \Delta \mathrm{Cq}}$ method (20). All quantities are expressed as fold changes relative to the expression of $\beta$-actin. The RT-qPCR reactions were performed in triplicate.

Statistical analysis. Values are expressed as the mean \pm standard deviation. The data were compared using the Mann-Whitney U-test, unpaired Student's t-test or one-way and mixed ANOVA followed by Bonferroni's post-hoc test. Data were analysed using GraphPad Prism version 6.0 (GraphPad Software, Inc.). $\mathrm{P}<0.05$ was considered to indicate a statistically significant difference.

\section{Results}

Locomotor deficits at day 28 of CIA. Baseline locomotion, including total active time, total immobility time, total movement distance and mean motion velocity, did not significantly differ between mice assigned to the normal group and those assigned to the model group (Fig. S2A). A total of 28 days after the first induction of CIA, locomotion was again assessed via the OFT. As expected, mice with CIA traveled shorter distances in the 5-min assessment but had a significantly higher total active time, resulting in a lower mean motion velocity compared with that of mice in the normal group (Fig. S2B). However, there were no locomotor differences between mice in the CIA group and those assigned to the UC-MSC treatment group (Fig. S2C-E).

Clinical arthritis and ankle thickness are unaffected by UC-MSC treatment. UC-MSCs are a potent treatment modality with beneficial effects in arthritis models and human patients with RA $(7,8)$. However, in the CIA model of the present study (Fig. S3A), treatment with a total of $15 \times 10^{6}$ UC-MSCs did not significantly affect the ankle thickness or arthritis scores compared with those of untreated mice with CIA (Fig. S3B-D).

Knee joint arthritis histopathology is unaffected by UC-MSC treatment. Joint inflammation and cartilage damage in the CIA model were examined via $\mathrm{HE}$ and toluidine blue staining, respectively. It was indicated that CIA resulted in synovitis and bone erosion (as detected by HE staining), as well as articular cartilage and proteoglycan loss (as indicated by toluidine blue 

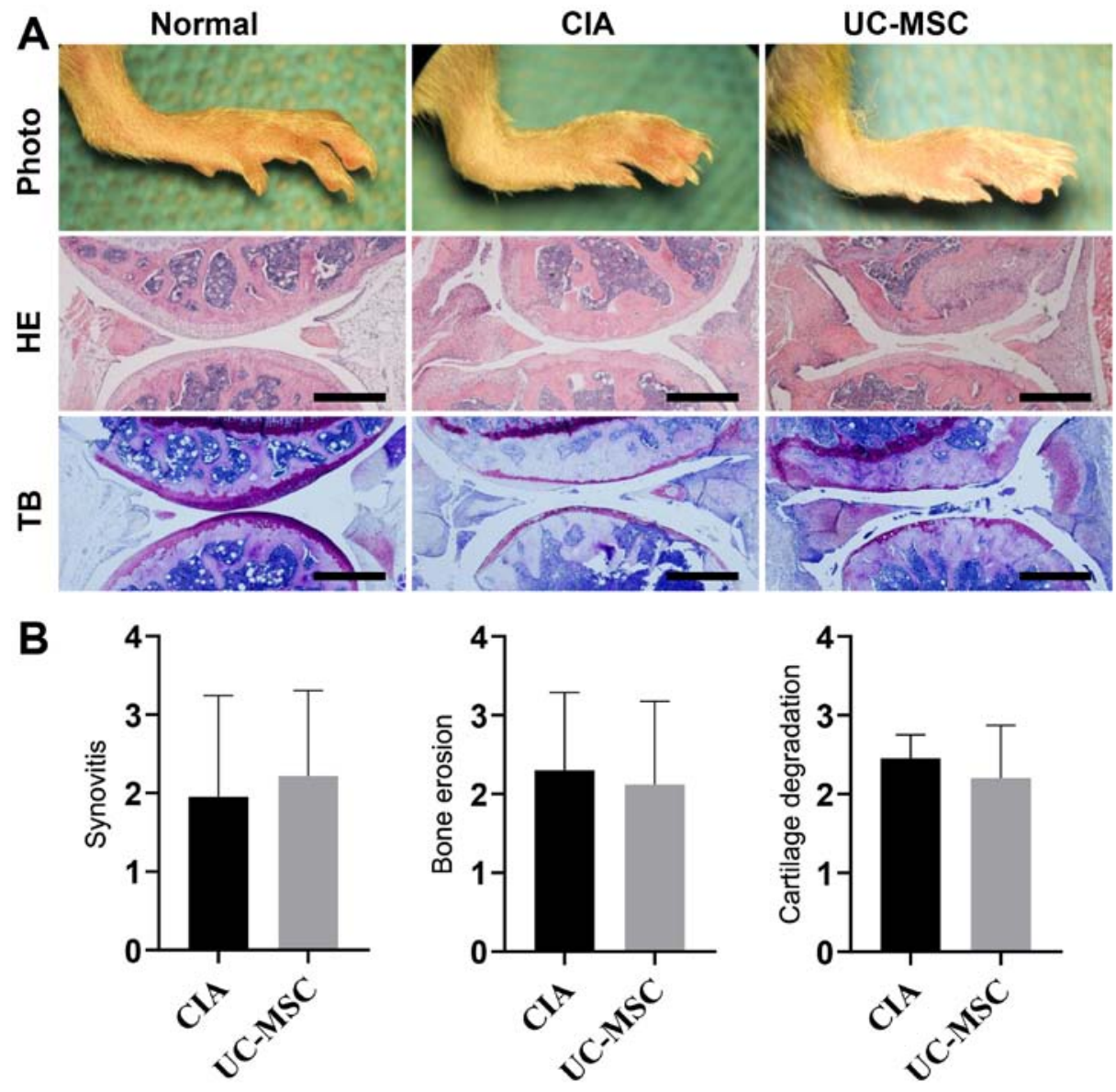

Figure 1. UC-MSC treatment does not ameliorate knee joint and hind limb pathology in mice with CIA. (A) Representative hind paw images and joint sections with HE and TB staining (scale bar, $200 \mu \mathrm{m}$ ). (B) Scoring of synovitis, bone erosion and cartilage degradation. Values are expressed as the mean \pm standard deviation $(\mathrm{n}=11)$. TB, toluidine blue; CIA, collagen-induced arthritis; UC-MSC, umbilical cord mesenchymal stem cell.

staining) in comparison with the normal group (Fig. 1A). However, the results in the group receiving UC-MSC treatment did not differ from the untreated CIA group. Furthermore, the histology was consistent with the observed increase in clinical arthritis scores and left/right paw swelling in mice with CIA, regardless of UC-MSC treatment. In addition, UC-MSC treatment did not decrease synovitis, bone erosion or cartilage degradation in mice with CIA (Fig. 1B).

Rheumatoid cachexia is unaffected by UC-MSC treatment. Mice with CIA exhibited a significant decrease in body weight at various points after the first immunization. However, there was no significant difference between the CIA group and the UC-MSC group (Fig. 2A). Although food intake did not differ among the groups (Fig. 2B), the normalized food intake (food intake/body weight) was higher in mice with CIA from day 42 onwards (there was no significance on day 77) (Fig. 2C). After the mice were sacrificed on day 84 , the gastrocnemius muscle weight and normalized gastrocnemius muscle weight (ratio of muscle weight to body weight) in the UC-MSC group were significantly lower compared with those in the CIA group, and a similar if insignificant trend was observed on the left (Fig. 2D and E).

Muscle fiber cross-sectional area is unaffected by UC-MSC treatment. Histological analysis of cross-sections of gastrocnemius muscles with HE staining revealed additional pathological features of CIA (Fig. 3A). The cross-sectional area of gastrocnemius muscle fibers was significantly decreased in mice with CIA, as well as in those treated with UC-MSCs (Fig. 3B). Of note, mice with CIA that were treated with frequent high doses of UC-MSCs did not exhibit any improved muscle fiber areas (Fig. 3C).

Proinflammatory and fibrosis cytokines in CIA. Inflammation and fibrosis may lead to changes in muscle weight $(17,18)$. As the gastrocnemius muscle weight was further decreased in CIA mice treated with UC-MSCs, the present study investigated whether UC-MSCs induce an inflammatory response. Immunostaining of tissue collected on day 84 was negative for markers of B cells (CD19), T cells (CD3 and CD4) and macrophages (iNOS and F4/80), indicating that there was no inflammatory-cell infiltration. However, RT-qPCR analyses identified significant increases in the expression levels of the inflammatory cytokines TNF- $\alpha$, IL- 6 and IL- $1 \beta$ in mice with CIA as compared with those in normal mice (Fig. 4). Furthermore, mice treated with UC-MSCs exhibited similar increases, except for the expression of $\mathrm{TNF}-\alpha$, which was significantly enhanced compared with that in untreated mice with CIA. There were neither any significant differences among the groups in the expression levels of iNOS, TGF- $\beta$, FN1 and $\alpha$-SMA, nor in the expression levels of fibrosis genes (COLIa1, COLIa2 and COLIIIa1) (Fig. S4). 
A

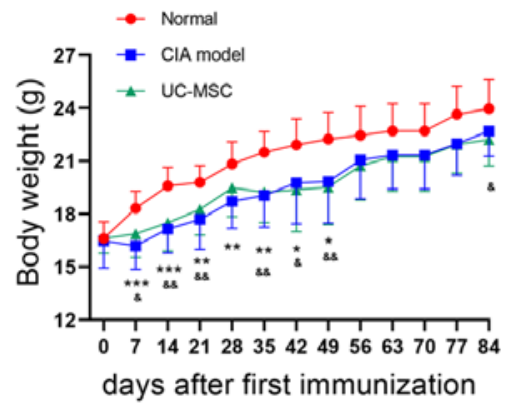

B

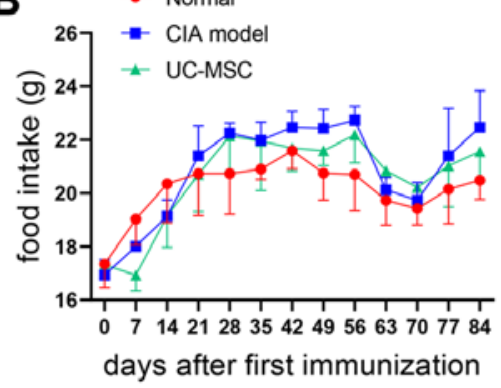

C

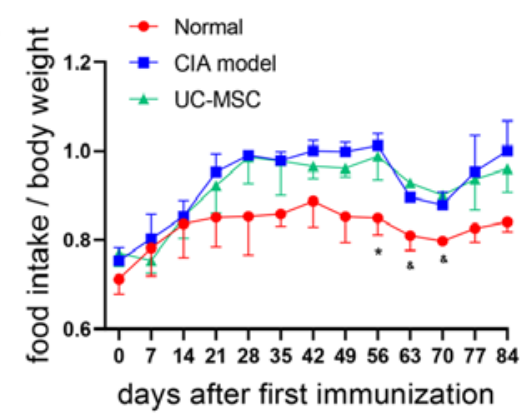

D

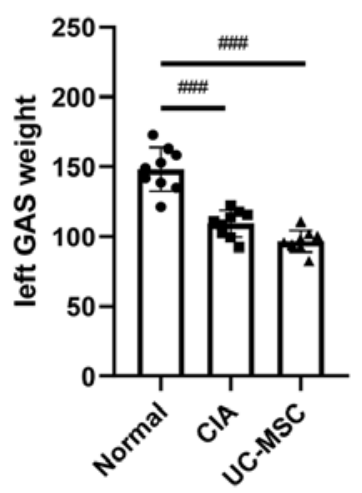

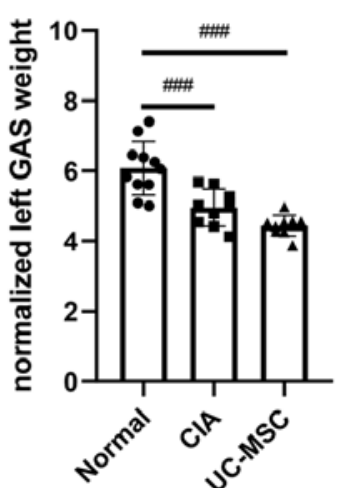

E

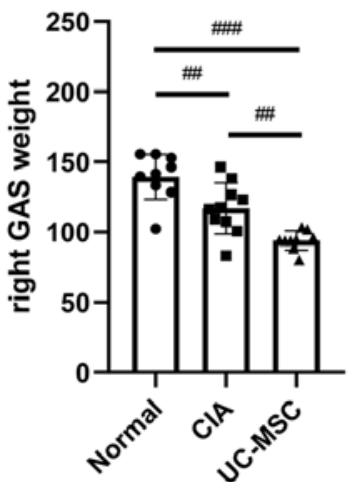

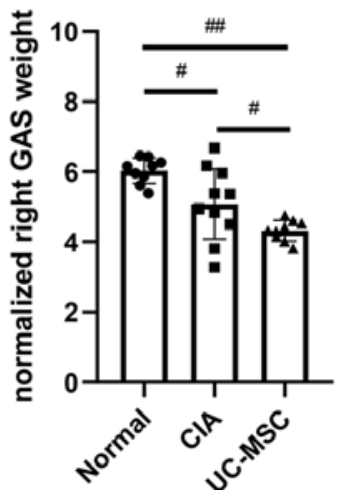

Figure 2. UC-MSCs do not ameliorate rheumatoid cachexia. (A) Body weights, (B) food intake and (C) normalized food intake in the normal group, CIA group and UC-MSC-treated group ( $\mathrm{n}=10-12)$. ${ }^{*} \mathrm{P}<0.05$, ${ }^{* *} \mathrm{P}<0.01$ and ${ }^{* * *} \mathrm{P}<0.001$ vs. CIA group; ${ }^{\circledR} \mathrm{P}<0.05$ and ${ }^{\&} \& \mathrm{P}<0.01$ vs. UC-MSC-treated group. Weights and normalized weights of (D) left and (E) right GAS. Values are presented as the mean \pm standard deviation ( $\mathrm{n}=10-12$ for panel $\mathrm{D} ; \mathrm{n}=9-10$ for panel $\mathrm{E}){ }^{\sharp} \mathrm{P}<0.05$, ${ }^{\# \#} \mathrm{P}<0.01,{ }^{\# \#} \mathrm{P}<0.001$. CIA, collagen-induced arthritis; UC-MSC, umbilical cord mesenchymal stem cell; GAS, gastrocnemius muscles.

A

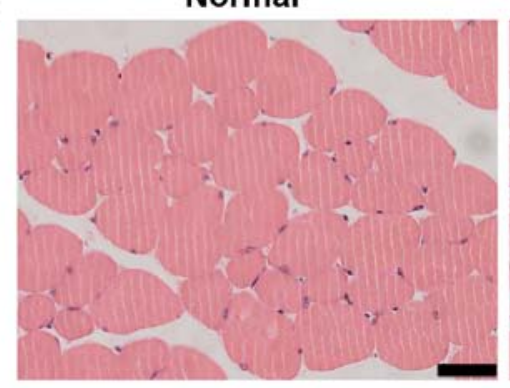

B

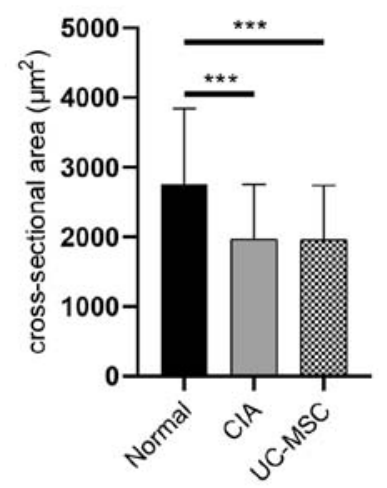

CIA group

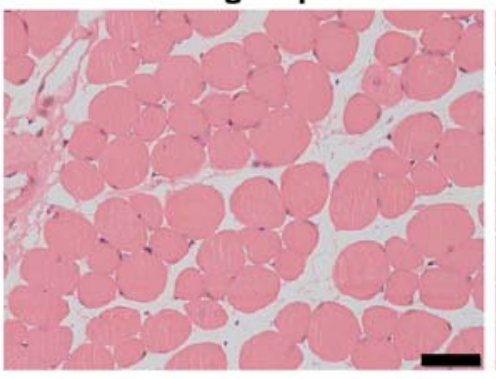

C

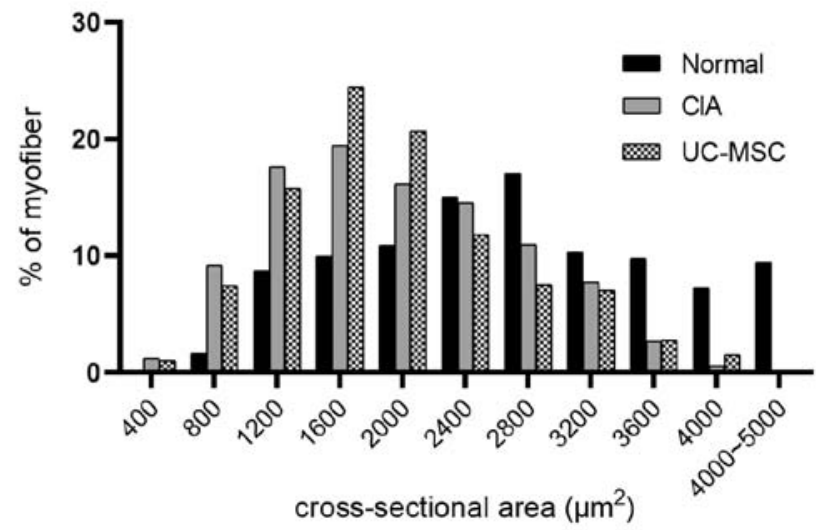

Figure 3. Reduced gastrocnemius cross-sectional area in mice with CIA. (A) Representative histopathology of gastrocnemius muscle fibers on day 84 (scale bar, $2 \mathrm{~mm}$ ). (B) Cross-sectional fiber areas of gastrocnemius muscles. Values are expressed as the mean \pm standard deviation ( $\mathrm{n}=6-8$; $100-200$ myofibers per mouse). ${ }^{* * *} \mathrm{P}<0.001$, as determined via one-way ANOVA. (C) Distributions of the cross-sectional areas of gastrocnemius muscle fibers ( $\mathrm{n}=10-11$; 100-200 myofibers per mouse). CIA, collagen-induced arthritis; UC-MSC, umbilical cord mesenchymal stem cell. 


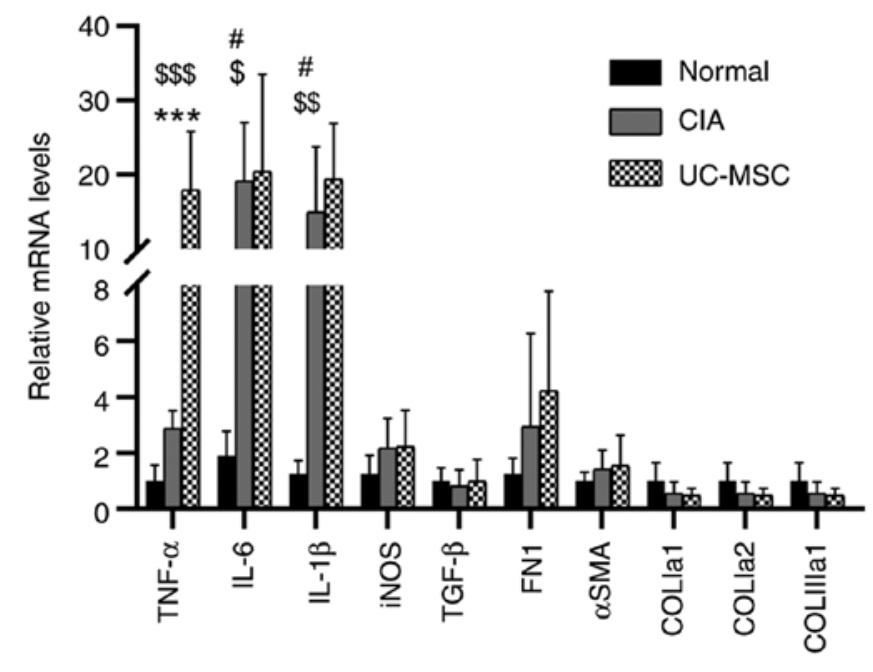

Figure 4. UC-MSC treatment does not decrease the immune response in the muscles of mice with CIA. Levels of relative mRNA expression were normalized to those of $\beta$-actin. Values are expressed as the mean \pm standard deviation ( $\mathrm{n}=6$-9). ${ }^{\#} \mathrm{P}<0.05$, normal vs. CIA; ${ }^{\$} \mathrm{P}<0.05,{ }^{\$ \$} \mathrm{P}<0.01,{ }^{\$ \$} \mathrm{P}<0.001$, normal vs. UC-MSC; ${ }^{* * *} \mathrm{P}<0.001$, CIA vs. UC-MSC. CIA, collagen-induced arthritis; UC-MSC, umbilical cord mesenchymal stem cell; iNOS, inducible nitric oxide synthase; SMA, smooth muscle actin; COLIa1, collagen type I a1 chain; COLIIIa1, collagen type III a1 chain; FN, fibronectin.

\section{Discussion}

MSCs, known for their immunomodulatory properties, have been reported to improve clinical symptoms and joint pathology (21-24). However, MSCs do not always exert immunoregulatory functions and may only promote immune responses under certain conditions (25). Specifically, the present study examined a novel cell-based therapeutic strategy for animal arthritis using high-frequency and -dose injection of UC-MSCs, which has so far remained largely elusive. The present study first addressed whether a high-frequency and -dose injection of UC-MSCs possessed anti-arthritis properties and the results demonstrated the inability of this treatment to exert a beneficial effect on clinical symptoms and joint protection in the experimental arthritis model. Next, the effect of high-frequency and -dose injection of UC-MSCs on muscle atrophy was assessed and the results indicated that this treatment decreased gastrocnemius muscle weight and increased the levels of TNF- $\alpha$ in this muscle. Therefore, the present UC-MSC therapy aggravated muscle wasting and had no significant effect on arthritis symptoms and body weight in an experimental model of RA.

RA is a systemic disease mainly affecting small joints. The treatment of RA usually comprises systemic administration of agents such as methotrexate and leflunomide, which may control disease activity of inflamed joints via immunoregulation (1). Local intraarticular injection is generally suitable for large joints and has the advantage of controlling the symptoms of the injected joint, with relatively fewer effects on the non-injected joints (26). Thus, due to the small joint cavity and poor maneuverability, local intraarticular injection is rarely used to treat RA. Based on the above considerations, intravenous administration of UC-MSCs was used in the present study. It was previously reported that the injected UC-MSCs preferentially migrated to the inflammatory joint sites of the rats as proven by UC-MSCs being labeled with superparamagnetic iron oxide nanoparticles and bromodeoxyuridine (27). In the present study, no staining for UC-MSC markers in the inflamed joints was performed after animal sacrifice, so it cannot be decisively proven whether all of the effects observed were induced by UC-MSCs directly. However, it was demonstrated that UC-MSCs are able to secrete a wide range of functional factors, including cytokines, chemokines and metabolites, which mostly have beneficial effects on RA due to their anti-inflammatory activity (7) and systemic immunomodulatory effects (9), suggesting that the therapeutic effect is not only entirely dependent on local effects of articular administration but also on systemic effects. It was previously reported that a single dose of $5 \times 10^{6}$ UC-MSCs was effective in relieving clinical symptoms in arthritis models (10). Furthermore, Liu et al (11) revealed that injections of $5 \times 10^{6}$ UC-MSC cells on days 28 and 56 improved joint damage and served a beneficial role in arthritis-related osteoporosis. However, there is an important balance between the pro-inflammatory and anti-inflammatory effects of the functional factors. It is well documented that MSCs fail to exhibit a therapeutic effect on RA $(28,29)$, and that intravenously infused MSCs induce inflammatory responses in vivo (30). The present study suggested that three weekly injections of $5 \times 10^{6}$ UC-MSCs did not relieve arthritis and muscle atrophy. The inconsistencies among previous studies and the present results may be associated with the stimulatory effects of the microenvironment on MSCs, which may hamper their immunosuppressive properties or even enhance the inflammatory process. Thus, it was suggested that the lack of therapeutic benefit may be due to the dose and frequency of UC-MSCs administered, which may have led to UC-MSCs losing their immunomodulatory properties and becoming pro-inflammatory.

Similar to RA, the CIA model is a chronic and progressive disease manifested by arthritis and muscle cachexia prior to day 45 (31) or day 65 (5). The molecular phenotype of muscle mass loss involves increases in inflammatory cytokines, including IL-1 $\beta$, IL-8, IL-6 and Toll-like receptor-4 (32). A study from another field of research reported that MSCs promoted muscle regeneration by enhancing angiogenesis (33). However, the effects of MSCs on RA muscle atrophy have remained largely elusive. The present study focused on the effects MSC interventions on muscle atrophy in a CIA model. The results demonstrated that body and muscle weight were reduced, while the levels of pro-inflammatory cytokines, including TNF- $\alpha$, were increased in gastrocnemius muscles after UC-MSC therapy, as detected via RT-qPCR. Although inflammation is an important driver of RA skeletal muscle disease, immune cells did not infiltrate into the muscle, which suggests that the skeletal cachexia of CIA was not due to systemic inflammation but rather the muscle cells themselves. A possible reason for this is that the UC-MSC group demonstrated more severe arthritis than the CIA group, meaning that animals moved less and the muscles wasted more. Furthermore, muscle fibrosis has been detected in skeletal cachexia in animal models of RA (19). The present study examined the effects of CIA up to 84 days after immunization but did not observe any fibrotic manifestations. 
The present study had certain limitations that are worth mentioning. It was previously suggested that syngeneic or allogeneic MSCs provided a clinical benefit when administered intravenously prior to the onset of arthritis (infused MSCs at days 1 and 5 or 4 and 9 post-Freund's complete adjuvant injection) in an adjuvant-induced arthritis rat model (29); thus, a study with earlier injection of UC-MSCs prior to arthritis onset should be designed. Furthermore, only one therapeutic dosage regimen was tested and further studies using different dose gradients in CIA models are required to investigate the optimal dose and frequency. Of note, the effectiveness of UC-MSC injections at a low frequency and dose have been demonstrated in previous studies. However, the present results indicated that frequent high-dose injections of UC-MSCs failed to exert anti-arthritis and anti-muscle cachexia effects.

In conclusion, skeletal muscle cachexia persisted even in the CIA mice at day 84. However, frequent injection of high-dose of UC-MSCs slightly aggravated arthritis and skeletal muscle cachexia in CIA mice.

\section{Acknowledgements}

Not applicable.

\section{Funding}

The present study was supported by the Science and Technology Department of Henan Province (grant no. 212102310200) and the National Natural Science Foundation of China (grant no. 81970312).

\section{Availability of data and materials}

The datasets used and/or analysed during the current study are available from the corresponding author on reasonable request.

\section{Authors' contributions}

LA, HY and ZZ contributed to the study design, data collection, statistical analysis, data interpretation and manuscript preparation. LA, TC, LW, SA, YL and $\mathrm{HH}$ performed the experiments and data curation. $\mathrm{LA}, \mathrm{HH}, \mathrm{ZZ}$ and $\mathrm{HY}$ performed manuscript editing and review. LA, ZZ and HY confirmed the authenticity of the raw data. All authors read and approved the final manuscript.

\section{Ethics approval and consent to participate}

Ethics approval for the human experiments was obtained from the Ethical Board of the Stem Cell Clinical Research Center of Henan Provincial People's Hospital [Zhengzhou, China; approval no. 1 (2019)]. The donor provided written informed consent. Ethics approval for the animal experiments was obtained from the Animal Ethics Committee of Peking University (Beijing, China; approval no. J201985).

\section{Patient consent for publication}

Not applicable.

\section{Competing interests}

The authors have declared that they have no competing interests.

\section{References}

1. Aletaha D and Smolen JS: Diagnosis and management of rheumatoid arthritis: A review. JAMA 320: 1360-1372, 2018.

2. Santo RCE, Fernandes KZ, Lora PS, Filippin LI and Xavier RM: Prevalence of rheumatoid cachexia in rheumatoid arthritis: A systematic review and meta-analysis. J Cachexia Sarcopenia Muscle 9: 816-825, 2018.

3. Cohen S, Nathan JA and Goldberg AL: Muscle wasting in disease: Molecular mechanisms and promising therapies. Nat Rev Drug Discov 14: 58-74, 2015.

4. Lin JZ, Liang JJ, Ma JD, Li QH, Mo YQ, Cheng WM, He XL, Li N, Cao MH, Xu D and Dai L: Myopenia is associated with joint damage in rheumatoid arthritis: A cross-sectional study. J Cachexia Sarcopenia Muscle 10: 355-367, 2019.

5. Alabarse PVG, Lora PS, Silva JMS, Santo RCE, Freitas EC, de Oliveira MS, Almeida AS, Immig M, Teixeira VON, Filippin LI and Xavier RM: Collagen-induced arthritis as an animal model of rheumatoid cachexia. J Cachexia Sarcopenia Muscle 9: 603-612, 2018.

6. Burmester GR and Pope JE: Novel treatment strategies in rheumatoid arthritis. Lancet 389: 2338-2348, 2017.

7. Leyendecker A Jr, Pinheiro CCG, Amano MT and Bueno DF: The use of human mesenchymal stem cells as therapeutic agents for the in vivo treatment of immune-related diseases: A systematic review. Front Immunol 9: 2056, 2018.

8. Liu L, Farhoodi HP, Han M, Liu G, Yu J, Nguyen L, Nguyen B, Nguyen A, Liao W and Zhao W: Preclinical evaluation of a single intravenous infusion of hUC-MSC (BX-U001) in rheumatoid arthritis. Cell Transplant 29: 963689720965896, 2020.

9. Shi Y, Wang Y, Li Q, Liu K, Hou J, Shao C and Wang Y: Immunoregulatory mechanisms of mesenchymal stem and stromal cells in inflammatory diseases. Nat Rev Nephrol 14: 493-507, 2018

10. Sun Y, Kong W, Huang S, Shi B, Zhang H, Chen W, Zhang H, Zhao C, Tang X, Yao G, et al: Comparable therapeutic potential of umbilical cord mesenchymal stem cells in collagen-induced arthritis to TNF inhibitor or anti-CD20 treatment. Clin Exp Rheumatol 35: 288-295, 2017.

11. Liu C, Zhang H, Tang X, Feng R, Yao G, Chen W, Li W, Liang J, Feng $X$ and Sun L: Mesenchymal stem cells promote the osteogenesis in collagen-induced arthritic mice through the inhibition of TNF- $\alpha$. Stem Cells Int 2018: 4069032, 2018.

12. Lu LL, Liu YJ, Yang SG, Zhao QJ, Wang X, Gong W, Han ZB, $\mathrm{Xu} \mathrm{ZS}, \mathrm{Lu} \mathrm{YX}$, Liu D, et al: Isolation and characterization of human umbilical cord mesenchymal stem cells with hematopoiesis-supportive function and other potentials. Haematologica 91: 1017-1026, 2006.

13. Mushahary D, Spittler A, Kasper C, Weber V and Charwat V: Isolation, cultivation, and characterization of human mesenchymal stem cells. Cytometry A 93: 19-31, 2018.

14. Dominici M, Le Blanc K, Mueller I, Slaper-Cortenbach I, Marini Fc, Krause Ds, Deans Rj, Keating A, Prockop Dj and Horwitz Em: Minimal criteria for defining multipotent mesenchymal stromal cells. The international society for cellular therapy position statement. Cytotherapy 8: 315-317, 2006.

15. Brand DD, Latham KA and Rosloniec EF: Collagen-induced arthritis. Nat Protoc 2: 1269-1275, 2007

16. Miyoshi M and Liu S: Collagen-induced arthritis models. Methods Mol Biol 1868: 3-7, 2018.

17. Kong X, Zhang Z, Fu T, Ji J, Yang J and Gu Z: TNF- $\alpha$ regulates microglial activation via the NF- $\kappa B$ signaling pathway in systemic lupus erythematosus with depression. Int J Biol Macromol 125: 892-900, 2019.

18. Brenner M, Meng HC, Yarlett NC, Griffiths MM, Remmers EF, Wilder RL and Gulko PS: The non-major histocompatibility complex quantitative trait locus Cia10 contains a major arthritis gene and regulates disease severity, pannus formation, and joint damage. Arthritis Rheum 52: 322-332, 2005.

19. Oyenihi AB, Ollewagen T, Myburgh KH, Powrie YSL and Smith C: Redox status and muscle pathology in rheumatoid arthritis: Insights from various rat hindlimb muscles. Oxid Med Cell Longev 2019: 2484678, 2019. 
20. An L, Li Z, Shi L, Wang L, Wang Y, Jin L, Shuai X and Li J: Inflammation-targeted celastrol nanodrug attenuates collagen-induced arthritis through NF- $\mathrm{KB}$ and Notch1 pathways. Nano Lett 20: 7728-7736, 2020.

21. Park KH, Mun CH, Kang MI, Lee SW, Lee SK and Park YB: Treatment of collagen-induced arthritis using immune modulatory properties of human mesenchymal stem cells. Cell Transplant 25: 1057-1072, 2016.

22. Park MJ, Lee SH, Moon SJ, Lee JA, Lee EJ, Kim EK, Park JS Lee J, Min JK, Kim SJ, et al: Overexpression of soluble RAGE in mesenchymal stem cells enhances their immunoregulatory potential for cellular therapy in autoimmune arthritis. Sci Rep 6: 35933, 2016.

23. Wu CC, Liu FL, Sytwu HK, Tsai CY and Chang DM: CD146+ mesenchymal stem cells display greater therapeutic potential than CD146-cells for treating collagen-induced arthritis in mice. Stem Cell Res Ther 7: 23, 2016.

24. Shu J, Pan L, Huang X, Wang P, Li H, He X and Cai Z: Transplantation of human amnion mesenchymal cells attenuates the disease development in rats with collagen-induced arthritis Clin Exp Rheumatol 33: 484-490, 2015.

25. Inoue S, Popp FC, Koehl GE, Piso P, Schlitt HJ, Geissler EK and Dahlke MH: Immunomodulatory effects of mesenchymal stem cells in a rat organ transplant model. Transplantation 81: 1589-1595, 2006.

26. Santos JM, Bárcia RN, Simões SI, Gaspar MM, Calado S, Agua-Doce A, Almeida SC,Almeida J,Filipe M, Teixeira M, et al: The role of human umbilical cord tissue-derived mesenchymal stromal cells (UCX®) in the treatment of inflammatory arthritis. J Transl Med 11: 18, 2013.

27. Gu J, Gu W, Lin C, Gu H, Wu W, Yin J, Ni J, Pei X, Sun M, Wang F, et al: Human umbilical cord mesenchymal stem cells improve the immune-associated inflammatory and prothrombotic state in collagen type-II-induced arthritic rats. Mol Med Rep 12: 7463-7470, 2015.
28. Luz-Crawford P, Torres MJ, Noël D, Fernandez A, Toupet K, Alcayaga-Miranda F, Tejedor G, Jorgensen C, Illanes SE, Figueroa FE, et al: The immunosuppressive signature of menstrual blood mesenchymal stem cells entails opposite effects on experimental arthritis and graft versus host diseases. Stem Cells 34: 456-469, 2016

29. Papadopoulou A, Yiangou M, Athanasiou E, Zogas N, Kaloyannidis P, Batsis I, Fassas A, Anagnostopoulos A and Yannaki E: Mesenchymal stem cells are conditionally therapeutic in preclinical models of rheumatoid arthritis. Ann Rheum Dis 71: 1733-1740, 2012.

30. Hoogduijn MJ, Roemeling-van Rhijn M, Engela AU, Korevaar SS Mensah FK, Franquesa M, de Bruin RW, Betjes MG, Weimar W and Baan CC: Mesenchymal stem cells induce an inflammatory response after intravenous infusion. Stem Cells Dev 22: 2825-2835, 2013.

31. Filippin LI, Teixeira VN, Viacava PR, Lora PS, Xavier LL and Xavier RM: Temporal development of muscle atrophy in murine model of arthritis is related to disease severity. J Cachexia Sarcopenia Muscle 4: 231-238, 2013.

32. Huffman KM, Jessee R, Andonian B, Davis BN, Narowski R, Huebner JL, Kraus VB, McCracken J, Gilmore BF, Tune KN, et al: Molecular alterations in skeletal muscle in rheumatoid arthritis are related to disease activity, physical inactivity, and disability. Arthritis Res Ther 19: 12, 2017.

33. Arutyunyan I, Fatkhudinov T, Kananykhina E, Usman N, Elchaninov A, Makarov A, Bolshakova G, Goldshtein D and Sukhikh G: Role of VEGF-A in angiogenesis promoted by umbilical cord-derived mesenchymal stromal/stem cells: In vitro study. Stem Cell Res Ther 7: 46, 2016. 